\title{
The impact of plaque type on strut embedment/protrusion and shear stress distribution in bioresorbable scaffold
}

\section{Ryo Torii ${ }^{1 \dagger}$, Erhan Tenekecioglu ${ }^{2 \dagger}$, Yuki Katagiri ${ }^{3}$, Ply Chichareon ${ }^{3}$, Yohei Sotomi ${ }^{3}$, Jouke Dijkstra ${ }^{4}$, Taku Asano ${ }^{3}$, Rodrigo Modolo ${ }^{3}$, Kuniaki Takahashi ${ }^{3}$, Hans Jonker ${ }^{5}$, Robert van Geuns ${ }^{2}$, Yoshinobu Onuma ${ }^{2}$, Kerem Pekkan ${ }^{6}$, Christos V. Bourantas ${ }^{7,8}$, and Patrick W. Serruys ${ }^{2,9 *}$}

\begin{abstract}
${ }^{1}$ Department of Mechanical Engineering, University College London, London, UK; ${ }^{2}$ Department of Interventional Cardiology, Erasmus University Medical Center, Thoraxcenter, Rotterdam, The Netherlands; ${ }^{3}$ Department of Cardiology, Academic Medical Center, University of Amsterdam, Amsterdam, The Netherlands; ${ }^{4}$ LKEB-Division of Image Processing, Department of Radiology, Leiden University Medical Center, Leiden, The Netherlands; ${ }^{5}$ Cardialysis, Rotterdam, The Netherlands; ${ }^{6}$ Department of Mechanical Engineering, Koc University, Istanbul, Turkey; ${ }^{7}$ Institute of Cardiovascular Science, University College London, London, UK; ${ }^{8}$ Department of Cardiology, Barts Heart Centre,
\end{abstract} London, UK; and ${ }^{9}$ Imperial College, London, UK

Received 22 November 2018; editorial decision 20 May 2019; accepted 22 May 2019; online publish-ahead-of-print 19 June 2019

Aims

Scaffold design and plaque characteristics influence implantation outcomes and local flow dynamics in treated coronary segments. Our aim is to assess the impact of strut embedment/protrusion of bioresorbable scaffold on local shear stress distribution in different atherosclerotic plaque types.

Methods and results

Conclusion Fifteen Absorb everolimus-eluting Bioresorbable Vascular Scaffolds were implanted in human epicardial coronary arteries. Optical coherence tomography (OCT) was performed post-scaffold implantation and strut embedment/ protrusion were analysed using a dedicated software. OCT data were fused with angiography to reconstruct 3D coronary anatomy. Blood flow simulation was performed and wall shear stress (WSS) was estimated in each scaffolded surface and the relationship between strut embedment/protrusion and WSS was evaluated. There were 9083 struts analysed. Ninety-seven percent of the struts $(n=8840)$ were well-apposed and $243(3 \%)$ were malapposed. At cross-section level $(n=1289)$, strut embedment was significantly increased in fibroatheromatous plaques $(76 \pm 48 \mu \mathrm{m})$ and decreased in fibrocalcific plaques $(35 \pm 52 \mu \mathrm{m})$. Compatible with strut embedment, WSS was significantly higher in lipid-rich fibroatheromatous plaques $(1.50 \pm 0.81 \mathrm{~Pa})$, whereas significantly decreased in fibrocalcified plaques $(1.05 \pm 0.91 \mathrm{~Pa})$. After categorization of WSS as low $(<1.0 \mathrm{~Pa})$ and normal/high WSS $(\geq 1.0 \mathrm{~Pa})$, the percent of low WSS in the plaque subgroups were $30.1 \%, 31.1 \%, 25.4 \%$, and $36.2 \%$ for non-diseased vessel wall, fibrous plaque, fibroatheromatous plaque, and fibrocalcific plaque, respectively $(P$-overall $<0.001)$.

The composition of the underlying plaque influences strut embedment which seems to have effect on WSS. The struts deeply embedded in lipid-rich fibroatheromas plaques resulted in higher WSS compared with the other plaque types.

Keywords atherosclerotic plaque $\bullet$ bioresorbable scaffold $\bullet$ strut embedment $\bullet$ strut protrusion $\bullet$ shear stress

\section{Introduction}

Temporary scaffolding of diseased vessels by bioresorbable scaffolds (BRSs) were introduced to overcome the limitations of the metallic stents. However, unprecedented relatively high thrombosis rates have raised significant concerns about the efficacy of this technology. ${ }^{1}$ Late loss in the Absorb BRS was significantly larger than in the metallic everolimus-eluting stent (EES) and device-oriented composite

* Corresponding author. Tel: +31 (010) 206 2828; Fax: +31 (010) 206 2844. E-mail: patrick.w.j.c.serruys@gmail.com

† The first two authors contributed as co-first author.

Published on behalf of the European Society of Cardiology. All rights reserved. @ The Author(s) 2019. For permissions, please email: journals.permissions@oup.com. 
endpoint (cardiac death, target vessel myocardial infarction, and clinically-indicated target lesion revascularization) at 3 years followup was higher in Absorb than in metallic Xience (10\% vs. $5 \%$, $P=0.043){ }^{1}$ In the meta-analysis of five randomized clinical trials ${ }^{2}$ comparing the Absorb and the EES has shown that post-procedural minimal lumen diameter was a predictive factor for device thrombosis which emphasizes the role of aggressive implantation strategy with high-pressure post-dilatation using intracoronary imaging techniques to optimize scaffold expansion. ${ }^{3}$ Intracoronary imaging guided BRS implantation assists in sizing the diseased vessel segment and reference vessel to avoid any mismatch between the diseased and healthy reference vessel segments. To achieve strut embedment without scaffold disruption and for accurate measurement of minimal scaffold area and scaffold asymmetry, intracoronary imaging guiding is indispensable. ${ }^{4}$

Stent/scaffold design has impact on the local flow dynamics in treated vessel segment. This effect depends not only on the device design but also the embedment/protrusion of the scaffold struts within the vessel wall. Well-embedded struts induce less flow disruptions with shorter flow separations, whereas increased strut protrusion may cause flow turbulence and recirculation zones around the struts. These disrupted flow areas yield low wall shear stress (WSS) triggering various pathobiological reactions in platelets and endothelial layer of the vessel wall that may result in excessive neointimal hyperplasia. ${ }^{5}$ The histomorphometric properties of the treated vessel segments, in particularly the underlying atherosclerotic plaque properties determine strut apposition and penetration status. ${ }^{6}$

In the present study, we investigated the strut embedment/protrusion patterns in different plaque types treated with BRS and its effect on the local WSS distribution in treated segments.

\section{Methods}

\section{Patient population and study device}

Fifteen patients implanted with an Absorb bioresorbable vascular scaffold (Absorb, Abbott Vascular, USA), from the Absorb Cohort B2 first-inman study, were included in the present analysis. The selection criteria for the cases were as follows; the OCT pullback should provide clear imaging of the vessel lumen with scaffold struts, the treated segments were relatively straight vessel segments to prevent any effect of curvatures on the shear stress alterations, and the cases should have two separate coronary angiograms with $>25^{\circ}$ angle between each other for a $3 \mathrm{D}$ centreline extraction to be used in vessel reconstruction for computational fluid dynamic (CFD) simulations. The Absorb is a polylactide scaffold eluting an antiproliferative drug everolimus, with two pairs of radiopaque markers at both ends of the scaffold.

\section{OCT image acquisition and data analysis}

OCT imaging was performed post-procedure in the treated coronary arteries using a frequency domain OCT system (C7-XR OCT Intravascular Imaging System; St. Jude Medical, St. Paul, MN, USA) that was pull-backed at a speed of $20 \mathrm{~mm} / \mathrm{s}$. A non-occlusive flushing technique was implemented during the pullback by injection of angiographic contrast medium for blood clearance.

The OCT data were analysed off-line using QCU-CMS software (Medis Medical Imaging systems, Leiden, The Netherlands) at every $200 \mu \mathrm{m}$ interval in the scaffolded segment and at every $400 \mu \mathrm{m}$ in the non-scaffolded segments. In scaffolded segment, in each OCT frame, the plaque composition was defined as following: Lipid tissue was delineated as a signal-poor region with poorly depicted borders and a fast drop-off in OCT signal, whereas calcium was defined as a signal-poor zone with sharply delineated borders and a gradual drop-off in OCT signal. OCT frames illustrating the segments with no or little intimal thickening and the typical three-layered structure of the intima, media, and adventitia were considered as normal vessel wall. Fibrous plaque was characterized as the tissue with a high backscattering and a relatively homogeneous OCT signal. Fibroatheroma was specified as a lipid-rich pool with circumferential extent $>90^{\circ}$ and fibrocalcific plaque, as a plaque that included calcific tissue and no lipid or lipid tissue with a circumferential extent $<90^{\circ}$. At 'strut-level' analysis, for each strut, the underlying tissue type was defined. At 'cross-section level', the predominant plaque type was determined when several plaque types were identified in one crosssection on OCT. ${ }^{7-9}$ The eccentricity index was calculated as the ratio between the minimal and the maximal diameter of each cross-section on OCT. ${ }^{10}$ Expansion index was defined in 'device-level' as minimum scaffold area divided by mean reference area."

Coronary angiograms were analysed using a semiautomated edge detection system (CAAS QCA-2D system, Pie Medical Imaging BV, Maastricht, the Netherlands) with the dye-filled catheter used for calibration purposes. In each scaffold, the largest balloon diameter at maximal inflation pressure during deployment or post-dilatation were recorded and used to calculate the balloon/artery ratio (defined as: mean inflated balloon diameter/mean reference vessel diameter). Acute absolute scaffold recoil was calculated as the difference between the mean diameter of the deployment/post-dilatation balloon in its highest pressure $(\mathrm{A})$ and the mean luminal diameter in the scaffolded segment at the end of the procedure (B). Acute percent scaffold recoil was defined as: A-B/A and expressed as percentage. ${ }^{11,12}$ Acute luminal gain was defined as the difference between pre- and post-procedural minimum lumen diameter, as assessed by quantitative coronary angiography $(\mathrm{QCA}) .^{13}$

\section{Embedment/protrusion analysis by OCT}

For strut embedment/protrusion analysis, the embedment depths and protrusion distances were measured semiautomatically implementing a dedicated version of the QCU-CMS software (version 4.69, Leiden University Medical Center, Leiden, The Netherlands). The embedment analysis in OCT was performed in the scaffolded segment at every $200 \mu \mathrm{m}$ longitudinal interval using the methodology described previously. ${ }^{8}$ Frames were excluded from stent strut measurement if $>30 \%$ of vessel wall was not visible, in which case the next frame was selected for analysis. Struts located at the ostium of a side-branch were excluded from the embedment analysis. Distances were adjusted based on the known thickness of the strut including the polymer coating. A scaffold strut was considered malapposed if the axial distance between strut surface and luminal surface was greater than the strut thickness including its polymer coating. The reproducibility of embedment analysis for Absorb was previously reported by Sotomi et al..$^{8}$

\section{Reconstruction of coronary artery anatomy}

Three-dimensional reconstruction of the treated coronary artery was performed using a validated methodology. ${ }^{14}$ The radiopaque markers and the anatomical landmarks (i.e. side branches), identified both on angiography and OCT, were used to define the segment of interest which included the scaffolded segment and $5 \mathrm{~mm}$ proximal and distal nonscaffolded edge segments. The OCT images demonstrating the segment of interest, were analysed at a $200 \mu \mathrm{m}$ interval in the scaffolded segment and $400 \mu \mathrm{m}$ interval in the remaining segment of interest. The flow area was defined by the luminal borders in native vessel segments. In the 
scaffolded segments, the adluminal side of the struts and the lumen borders in the inter-strut areas delineated the flow area. ${ }^{15,16}$ In two orthogonal $\left(>25^{\circ}\right)$ angiograms, the luminal borders and lumen centreline were extracted and the lumen centrelines were used to generate 3D luminal centreline to be the backbone of the segments of interest. ${ }^{14}$ The flow area contours detected in OCT were mounted perpendicularly onto the luminal centreline and anatomical landmarks (side-branches) were used to estimate their orientation. ${ }^{14}$

\section{Blood flow simulation}

CFD techniques were implemented to process 3D models. A finite volume mesh was generated and numerical blood flow simulation was performed. The WSS was estimated by solving the 3D Navier-Stokes equations (ANSYS Fluent, Canonsburg, PA, USA). ${ }^{17}$ To examine the influence of scaffold design on the local haemodynamic forces, the mesh density around the struts and at flow boundary near the vessel wall between the struts was increased so as to have average element edge of $30 \mu \mathrm{m}$ (equals to $1 / 5$ of the strut thickness). Blood was treated as a homogeneous, Newtonian fluid with a viscosity of $0.0035 \mathrm{~Pa} \cdot \mathrm{s}$ and a density of $1050 \mathrm{~kg} / \mathrm{m}^{3}$. A steady flow profile was implemented at the inflow of the $3 \mathrm{D}$ models. Blood flow for each reconstruction was estimated by measuring, in two angiographic projections, the number of frames required for the contrast agent to pass from inlet to the outlet of the reconstructed segment, the volume of the reconstructed segment and the cine frame-rate. ${ }^{17}$ The arterial wall was considered to be rigid and no-slip conditions were imposed at the scaffold surface and the reconstructed luminal surface. At the outlet of the model, zero pressure condition was implemented. WSS was calculated as the product of blood viscosity and the gradient of blood velocity at the wall and strut surface. WSS was measured in the native and the scaffolded segment around the circumference of the lumen per $5^{\circ}$ interval (sector) and along the axial direction per $200 \mu \mathrm{m}$ interval with the use of an inhouse algorithm. ${ }^{18,19}$

\section{Statistical analysis}

Numerical data are expressed as mean \pm standard deviation or median and interquartile range depending on their distribution which was tested by Kolmogorov-Smirnov test. Continuous variables with normal and non-normal distributions were compared using the Student's $t$-tests and the Mann-Whitney $U$ tests, respectively. Categorical variables were compared using the Pearson's $\chi^{2}$ test or Fischer's exact test, as appropriate. As the data in the study have multilevel structure and unbalanced design, mixed linear model was used for the comparisons of continuous variables in the cross-section level analysis; the model took into account the clustered nature of $>1$ cross-sections from the same scaffold and $>1$ struts from the same cross-section, which might result in unknown correlations among measurements within the clusters. For WSS comparison between the plaque types, the multilevel model was built with fixed-effects on cross-sectional lumen area, embedment and protrusion distances with random effects on patient ID and cross-section ID. All statistical tests were two-tailed, and an $\alpha$-level of 0.05 was used to determine statistical significance. Analyses were performed using the statistical analysis programme SPSS V.23 (SPSS Inc., Chicago, IL, USA).

\section{Results}

Fourteen patients (15 lesions: 9 left anterior descending coronary arteries, 2 left circumflex and 4 right coronary arteries) were investigated in the present study. All the study patients were treated with a $3.0 \times 18 \mathrm{~mm}$ Absorb scaffold. Pre-dilatation was mandatory in
Table I Baseline characteristics of the studied population $(N=14$, lesion $=15)$

\begin{tabular}{lc}
\hline Age (years) & $\mathbf{6 1} \pm \mathbf{5}$ \\
\hline Male & $9(64)$ \\
Hypertension & $9(64)$ \\
Hypercholesterolaemia & $11(79)$ \\
Diabetes mellitus & $0(0)$ \\
Current smoking & $5(36)$ \\
Prior percutaneous coronary intervention & $2(14)$ \\
Prior myocardial infarction & $2(14)$ \\
Stable angina & $11(79)$ \\
Unstable angina & $1(7)$ \\
Silent ischaemia & $0(0)$ \\
Treated vessel & \\
$\quad$ Left anterior descending artery & $9(60)$ \\
Left circumflex artery & $2(13)$ \\
Right coronary artery & $4(27)$ \\
Ramus intermedius & $0(0)$ \\
\hline
\end{tabular}

Values are expressed as mean \pm standard deviation or $n(\%)$.

Table 2 Procedural characteristics

\begin{tabular}{lc}
\hline & $\mathbf{N}=\mathbf{1 4}, \mathbf{L}=\mathbf{1 5}$ \\
\hline ACC/AHA lesion class & \\
A & $0 \%(0)$ \\
B1 & $67 \%(10)$ \\
B2 & $33 \%(5)$ \\
Pre-dilatation & $0 \%(0)$ \\
Pre-dilatation pressure (atm) & $100 \%(15 / 15)$ \\
Diameter of scaffolds (mm) & $11.67 \pm 2.51$ \\
Expected scaffold diameter $(\mathrm{mm})$ & $3.00 \pm 0.0$ \\
Total length of study devices $(\mathrm{mm})$ & $3.30 \pm 0.11$ \\
Nominal scaffold area $\left(\mathrm{mm}{ }^{2}\right)$ & $18.0 \pm 0.0$ \\
Expected scaffold area $\left(\mathrm{mm}^{2}\right)$ & $7.07 \pm 0.0$ \\
Deployment pressure $(\mathrm{atm})$ & $8.54 \pm 0.58$ \\
Post-dilatation & $13.00 \pm 3.01$ \\
Post-dilatation pressure $(\mathrm{atm})$ & $60 \%(9)$ \\
Procedure complication & $18.29 \pm 5.06$ \\
Clinical device success & $13 \%(2)$ \\
Clinical procedure success & $100 \%(15 / 15)$ \\
\hline
\end{tabular}

Absorb Cohort-B trial and performed in all cases. Post-dilatation was left to operator's discretion in the study protocol. ${ }^{16}$ Patient characteristics are shown in Table 1. Procedural characteristics are demonstrated in Table 2. QCA analysis post-implantation showed an acute gain of $1.19 \pm 0.33 \mathrm{~mm}$ and acute percent recoil (using mean lumen diameter) of $7.6 \pm 6.5 \%$. Pre-implantation and post-implantation QCA data are shown in Table 3. 
Table 3 Results of QCA analysis pre-procedural,
post-procedural, and at 5-year follow-up

\section{Pre-procedure}

Lesion length $(\mathrm{mm})$

$9.81 \pm 3.87$

Pre-procedure reference vessel diameter $(\mathrm{mm})$

$2.56 \pm 0.31$

Pre-procedure minimum lumen diameter $(\mathrm{mm})$

$1.04 \pm 0.24$

Pre-procedure percent diameter stenosis (\%DS)

$58.91 \pm 10.19$

$D_{\max }$ proximal $(\mathrm{mm})$

$2.84 \pm 0.30$

$D_{\text {max }} \operatorname{distal}(\mathrm{mm})$

$2.69 \pm 0.29$

\section{Post-procedure}

Mean lumen diameter, in-scaffold $(\mathrm{mm})$

$2.63 \pm 0.22$

Reference lumen diameter $(\mathrm{mm})$

$2.62 \pm 0.23$

Minimum lumen diameter, in-scaffold ( $\mathrm{mm}$ )

$2.23 \pm 0.19$

In-scaffold percent diameter stenosis (\%DS)

$15.09 \pm 5.31$

In-scaffold acute absolute gain ( $\mathrm{mm}$ )

$1.18 \pm 0.31$

In-scaffold acute percent gain (\%)

$43.71 \pm 11.49$

In-scaffold acute absolute recoil (using mean

$0.21 \pm 0.18$

lumen diameter) (mm)

In-scaffold acute percent recoil (using mean

$7.64 \pm 6.49$

lumen diameter) (\%)

In-scaffold acute absolute recoil (using minimum

$0.24 \pm 0.17$

lumen diameter) ( $\mathrm{mm}$ )

In-scaffold acute percent recoil (using minimum

$9.69 \pm 6.88$

lumen diameter) (\%)

Ratio of post-dilatation balloon nominal diameter

$1.14 \pm 0.08$

to mean reference diameter

Pre-dilatation balloon diameter RVD ratio

$1.04 \pm 0.15$

\section{OCT analysis results}

OCT results are summarized in Table 4. Ninety-seven percent of the struts were well-apposed $(n=8840)$ to the vessel wall. There were 243 malapposed struts (3\%). Post-implantation expansion-index was $0.98 \pm 0.21$. Post-implantation mean scaffold area was $7.54 \pm 0.93 \mathrm{~mm}^{2}$ and in-scaffold mean lumen area was $7.43 \pm 0.88 \mathrm{~mm}^{2}$, whereas mean lumen area in proximal non-scaffolded edge segment was $7.50 \pm 1.84 \mathrm{~mm}^{2}$ and mean luminal area in distal non-scaffolded edge segment was $5.39 \pm 1.62 \mathrm{~mm}^{2}$. Mean eccentricity index was $0.67 \pm 0.053$. Cross-section level analysis $(n=1289)$ showed that in scaffolded segment, there is an inverse correlation between the median WSS and the eccentricity index $(r .-0.363, P<0.0001)$.

\section{Embedment/protrusion results}

At device level analysis, the strut protrusion distance was $113 \pm 14 \mu \mathrm{m}$. At cross-section level analysis, the protrusion distance was $115 \pm 42 \mu \mathrm{m}$. There was a significant relationship between the embedment depths and plaque types (Table 5, Figure 1). Struts over the fibroatheromatous plaques, were significantly deeper embedded than in other plaque types (Figure 2). Deployment/post-dilatation balloon pressures were found to have a modest effect on embedment depths $(r=0.28, P=0.048)$. Similar to the embedment analysis, the protrusion distances were also significantly related to the underlying plaque types. Lowest strut protrusion was noted in lipid-rich fibroatheromas, whereas in fibrous and fibrocalcific plaques, the strut

Table 4 Results of post-procedural OCT analysis

\begin{tabular}{lr}
\hline Post-procedural & $3.06 \pm 0.21$ \\
In-scaffold mean lumen diameter $(\mathrm{mm})$ & $2.75 \pm 0.22$ \\
In-scaffold minimum lumen diameter $(\mathrm{mm})$ & $7.43 \pm 0.88$ \\
In-scaffold mean lumen area $\left(\mathrm{mm}^{2}\right)$ & $5.98 \pm 0.96$ \\
In-scaffold minimum lumen area $\left(\mathrm{mm}^{2}\right)$ & $3.09 \pm 0.19$ \\
Mean scaffold diameter $(\mathrm{mm})$ & $2.80 \pm 0.21$ \\
Minimum scaffold diameter $(\mathrm{mm})$ & $7.54 \pm 0.93$ \\
Mean scaffold area $\left(\mathrm{mm}^{2}\right)$ & $6.19 \pm 0.93$ \\
Minimum scaffold area & $15.09 \pm 5.31$ \\
Post-procedure in-scaffold percent diameter & \\
$\quad$ stenosis (\%DS) & $0.20 \pm 0.03$ \\
Mean strut area (mm $\left.{ }^{2}\right)$ & $4.68 \pm 0.32$ \\
Mean lumen diameter in proximal edge segment $(\mathrm{mm})$ & $7.50 \pm 1.84$ \\
Mean lumen area in proximal edge segment $\left(\mathrm{mm}^{2}\right)$ & $4.03 \pm 1.23$ \\
Mean lumen diameter in distal edge segment $\left(\mathrm{mm}^{2}\right)$ & $5.39 \pm 1.62$ \\
Mean lumen area in distal edge segment $\left(\mathrm{mm}^{2}\right)$ & $0.67 \pm 0.05$ \\
Eccentricity index & $0.23 \pm 0.09$ \\
Asymmetry index & $0.98 \pm 0.21$ \\
\hline Expansion index &
\end{tabular}

protrusion was relatively higher (Table 5). The majority of the malapposed struts $(75 \%, n=149)$ were detected in the fibrocalcific group and the rest $(n=94)$ were in fibrous plaque group. All of the malapposed struts were detected in one case including totally 26 cross-sections. The newly developed thrombus post-implantation attached to the BVS struts without limiting the embedment analysis on OCT. Balloon sizing and inflation pressure had no direct effect on strut embedment, although high-pressure post-dilatation ( $\geq 20 \mathrm{~atm}$ ) resulted in numerically deeper embedment than low-pressure postdilatation. Balloon type did not present any significant effect on strut embedment at pre-dilatation $(P=0.49)$ or post-dilatation $(P=0.25)$.

\section{WSS results}

CFD results demonstrated higher WSS in fibroatheromas in Absorb (Figure 3). The lowest WSS values were documented in fibrous and fibrocalcific plaques (Table 5). After classifying the WSS as low $(<1.0 \mathrm{~Pa})$ and normal/high WSS $(\geq 1.0 \mathrm{~Pa})$, the percentages of low WSS in different plaque groups were $30.1 \%, 31.1 \%, 25.4 \%$, and $36.2 \%$ for the healthy vessel wall, the fibrous plaque, the fibroatheromatous plaque, and the fibrocalcific plaque, respectively ( $P$-overall $<0.001)$. Overall, there was an inverse linear relationship between strut protrusion distance and WSS (Figure 4). In all plaque types, there was a negative correlation between strut protrusion and WSS, which was pronounced in calcified plaques $(r=-0.240, P<0.01$ in fibrous; $r=-0.218, P<0.01$ in fibroatheroma and $r=-0.261, P<0.01$ in fibrocalcific plaques).

\section{Discussion}

The main results of our study can be summarized as follows: (i) Strut embedment/protrusion differed according to the underlying plaque 
Table 5 Cross-section level embedment/protrusion and WSS according to the plaque type

\begin{tabular}{|c|c|c|c|}
\hline Plaque type & Embedment depth $(\mu \mathrm{m})$ & Protrusion distance $(\mu \mathrm{m})$ & WSS (Pa) \\
\hline Non-atherosclerotic intimal thickening/normal vessel wall $(n=2275)$ & $47 \pm 34^{*, \Delta, *}$ & $123 \pm 34^{\pi, \%, \pi}$ & $1.44 \pm 0.9^{\theta, \S, \epsilon}$ \\
\hline Fibrous $(n=4191)$ & $53 \pm 40^{*, \#, \&}$ & $118 \pm 38^{\pi, \psi, \ddagger}$ & $1.24 \pm 0.78^{\alpha, \theta, \infty}$ \\
\hline Fibroatheromatous $(n=2027)$ & $76 \pm 48^{\#, \oint, \Delta}$ & $94.6 \pm 46^{t, \psi, \pi}$ & $1.50 \pm 0.81^{\Sigma, \S, \alpha}$ \\
\hline Fibrocalcific $(n=590)$ & $35 \pm 52^{\mathbb{R}, \oint, *}$ & $139 \pm 50^{\ddagger},+, Ж$ & $1.05 \pm 0.91^{\infty, \boldsymbol{\epsilon}, \Sigma}$ \\
\hline
\end{tabular}

For embedment: $* P=0.09,{ }^{\sharp} P<0.001,{ }^{\circledR} P<0.001,{ }^{\Phi} P<0.0001,{ }^{\Delta} P<0.0001,{ }^{\sharp} P<0.0001$.

For protrusion: ${ }^{\top} P=0.74,{ }^{W_{P}}<0.0001,{ }^{\pi} P<0.0001,{ }^{4} P<0.0001,{ }^{\ddagger} P<0.0001,{ }^{\dagger} P<0.0001$.

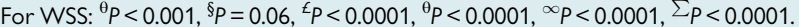

$n=$ total strut number in each plaque type; $P$-values come from mixed-effects regression analysis.
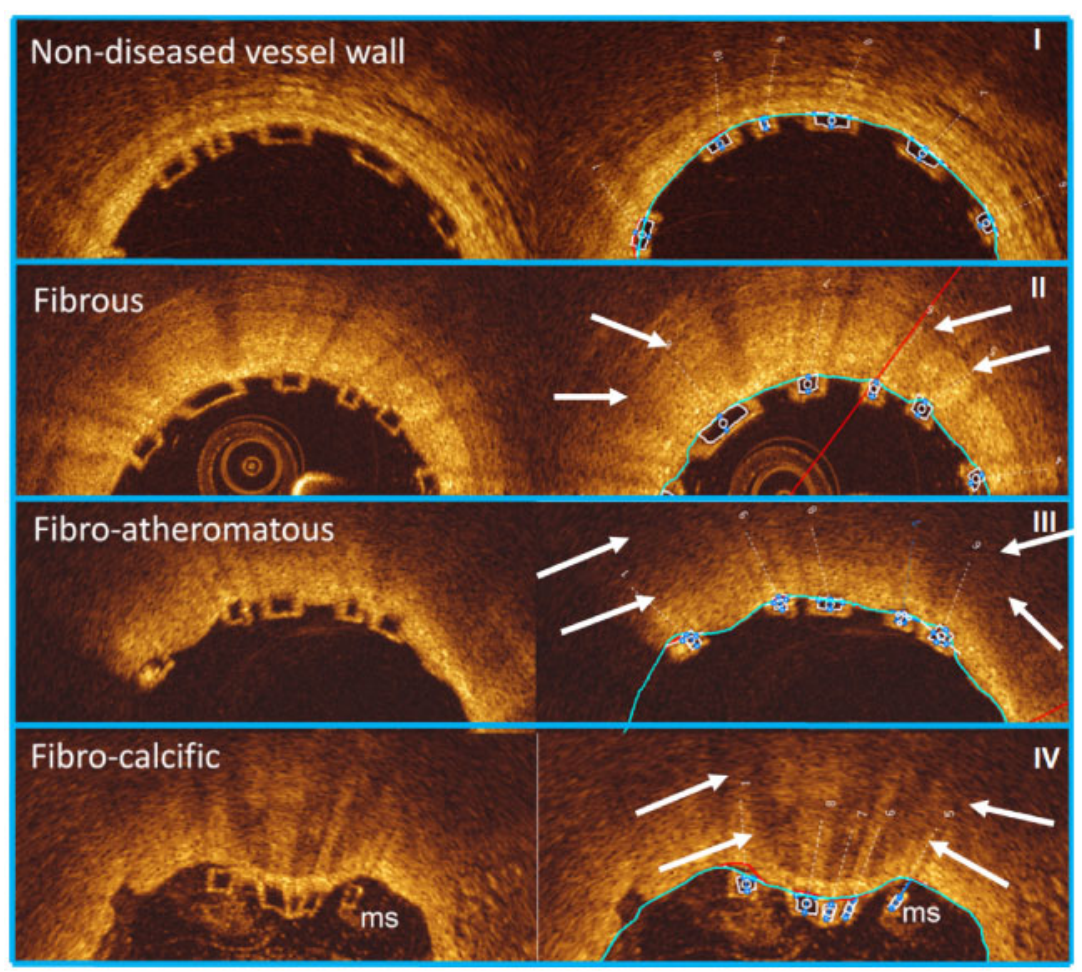

Figure I Rectangular shaped struts of Absorb BVS were automatically detected by QCU-CMS (v.14.9) after automatic detection of interpolated luminal contour (blue contour represents embedment contour), protrusion/embedment distances were analysed semiautomatically using the methodology described by Sotomi et al. ${ }^{8}$ The white arrows show the fibrous tissue in panel II, the lipid pool in panel III, and the calcified plaque in panel IV. The struts were well-embedded in fibroatheromatous plaques, whereas fibrocalcific plaque prevents deep penetration of the struts. Most of the malapposed struts (ms) were detected in vessel segments with calcified plaques.

type with an increased strut embedment in fibroatheromatous plaques; (ii) The strut embedment/protrusion pattern had an evident impact on WSS distribution: in fibroatheromas, where the struts were deeply embedded, there were less flow disruptions resulting in relatively higher WSS values compared with other plaque types; (iii) There was an inverse linear relationship between strut protrusion and WSS which was prominent in calcified plaques compared with the other plaque types.

Despite breathtaking advances in stent/scaffold designs over the last decades, restenosis and stent/scaffold thrombosis continue to be the
'Achilles heel' of the percutaneous coronary intervention (PCl). ${ }^{5} \mathrm{PCl}$ outcomes depend on several factors including implantation techniques, treated vessel segment features, particularly plaque characteristics and the design properties of the stent/scaffold. While the normal vessel wall is incompressible, the diseased vessel wall, including atherosclerotic plaque, is compressible and exhibits viscoelasticity while subjected to a pressure load. ${ }^{20,21}$ Based on this fact, stent/scaffold strut design and plaque type seem to be the main factors for strut penetration which determines local flow patterns, regulates neointimal hyperplasia and may be coupled with thrombus formation. ${ }^{5}$ 


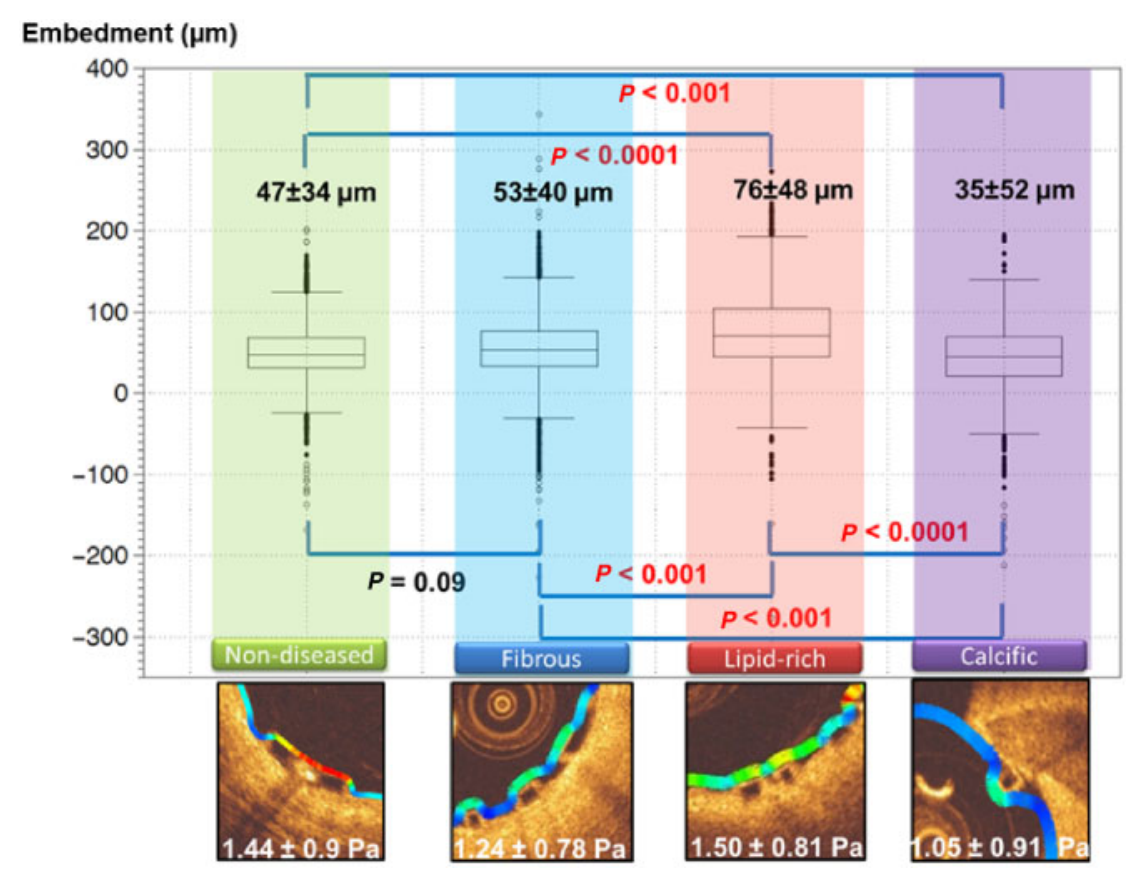

Figure 2 In the illustration, embedment distances were demonstrated for each group. Mean WSS for each group was shown in the lower OCT cross-section panels. $P$-values come from mixed effects analysis for the comparison of embedment distances between plaque types. In lipid-rich plaques, the struts embedment was deeper than in other plaque types.

\section{Plaque type and strut embedment/ protrusion impact on local shear stress}

The composition and morphology of the plaque determine its mechanical behaviours. The cellularity level and the tissue composition are the main determinants of the stiffness of the atherosclerotic plaques. Hypocellular plaques, such as fibrous and fibrocalcific plaques, are one to two times stiffer than the cellular lipid-rich fibroatheromatous plaques lipid-rich fibroatheromatous plaque is the most compliant and least stiff plaque type. ${ }^{22,23}$ Mechanical testing of the human atherosclerotic plaques unravelled that during compression-relaxation cycles, fibrous and calcified plaques behave similarly and are stiffer than the lipid-rich atheromatous plaques. ${ }^{24}$ Young's modulus (elastic modulus), indicates the resistance of a material to elastic deformation of fibrotic plaques are two-times higher than lipid-rich fibroatheromas. ${ }^{25}$

Stent/scaffold implantation imperils the vessel wall to higher stresses that may injure the internal elastic lamina, induces smooth muscle cell proliferation and neointimal tissue growth which may conclude with in-stent/scaffold restenosis. The level of vessel wall injury depends on stent/scaffold design, vessel geometry, curvature, implantation pressures during the procedure and biomechanical properties of the treated plaque. Under compressive load, particularly lipid-rich plaques demonstrate viscoelastic behaviour that reflects their relatively higher compliance. Furthermore, lipid-rich fibroatheromatous plaques are less stiffer than non-diseased healthy vessel wall. ${ }^{26}$ The stiffness of the vessel wall increases from lipid-rich atheromas to fibrotic and fibrocalcific lesions. ${ }^{27,28}$ In the present study, as expected, the polymeric struts penetrated deeper in lipid-rich atheromatous plaques than fibrous and fibrocalcific plaques, due to the viscoelastic properties of these plaque types.

Vessel wall stiffness, determined by plaque phenotype, influences scaffold expansion which has a potential impact on $\mathrm{PCl}$ outcomes. ${ }^{9}$ In calcified lesions, vessel expansion is less than non-calcified lesions. ${ }^{29}$ During implantation, in calcified, stiff lesions, the presence of calcium reduces the applied stress within the vessel wall that behaves as an 'absorber' protecting the vessel wall confronting high pressures. ${ }^{30}$ Implementing high pressures on cellular and compliant plaques may cause immense injury in the vessel wall, whereas the protective role of calcified plaque allows vessel dilatation at higher balloon pressures more safely. ${ }^{31}$ Therefore, the same device with the same applied pressures in different types of plaques will result in different levels of tissue injury and luminal gain. ${ }^{23}$ In the present study, after adjusting according to the plaque type, embedment/protrusion was slightly related with deployment pressures. ${ }^{9}$

The endothelization of the denuded artery wall and strut surfaces are related with local WSS. ${ }^{32}$ Low WSS induces neointimal growth that covers the struts and inter-strut vessel wall area. ${ }^{18,33}$ Low shear stress at the bottom beside the struts induces platelets and endothelium aggregation in the disrupted flow zones and trigger several biological pathways for neointimal regeneration and thrombus formation in the inter-strut zones. ${ }^{34}$ Flow separation and flow stagnation zones induce confluent endothelial cells migration away from the flow reattachment points demonstrated in flow chamber experiments. ${ }^{35}$ Higher embedment with less protrusion provides less flow disruption, yielded by non-low WSS in the vicinity of the struts that protects the vessel wall biology. ${ }^{36}$ Lipid-rich fibroatheromas with 

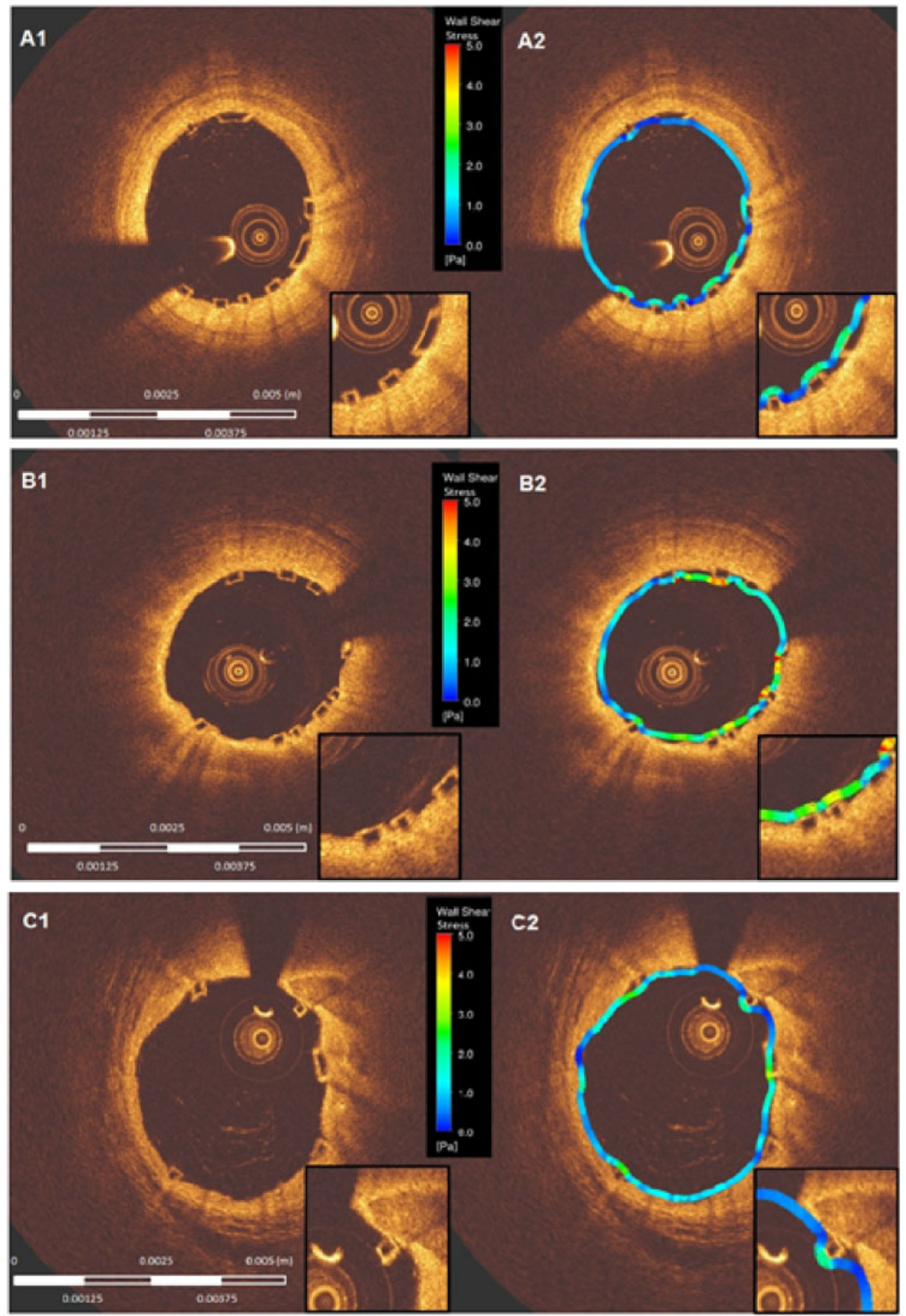

Figure 3 Representative OCT cross sections (left panels) with WSS contour overlay (right panels). Fibrous plaques were associated with higher strut protrusion distances that induced very low WSS (shown by the color contour overlay) due to flow obstruction and the formation of recirculation zones (A1, A2). Lipid-rich fibroatheromatous plaques allowed deeper strut embedment which induced less flow disruption in the vicinity of the struts resulting in low WSS gradient between top of the struts and inter-strut zones (B1, B2). Due to poor penetration in non-compliant calcified plaques, flow disruption induced higher gradients between the top of the struts and inter-strut zones (C1, C2). Small insert in each panel shows close-up view around struts.

deeper embedded struts demonstrated more 'favourable' WSS magnitudes, whereas stiffer fibrous and fibrocalcific plaques unravelled relatively lower WSS due to the flow disturbances related to less strut embedment. Well-embedded struts may reduce area with disturbed 'atheroprone' low WSS which might have favourable effects on vessel wall healing at follow-up post-implantation. 


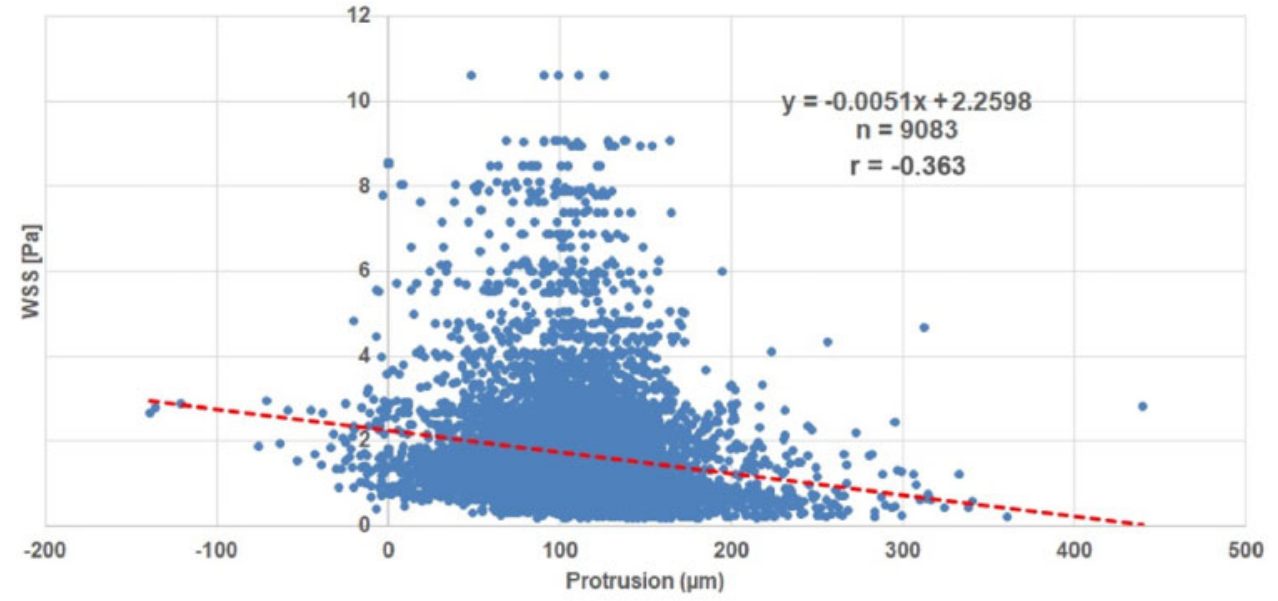

Figure 4 There was an inverse linear correlation between the median WSS and strut protrusion distances.

\section{Practical implications}

The idea of designing lesion specific stent and implantation techniques using intravascular imaging modalities has been a research subject. Due to its higher strut thickness, Absorb potentially induces flow disruptions more than the stents/scaffolds with thinner strut designs. ${ }^{37,38}$ However, in case of well-embedment, as in lipid-rich plaques, Absorb may provide more 'favourable' shear stress distribution due to deeper strut penetration which may potentially promote a strategy of BRS implantation according to the underlying plaque type and may mitigate the negative aspects of thicker strut and nonstreamline strut geometry. ${ }^{39}$

\section{Limitations}

The main limitation of the present study was low case number. Several criteria were implemented for filtering suitable cases. To prevent any effect of swirling-flow due to vessel curvature, on the scaffolded segment WSS distribution, we didn't include the cases with curvature and the cases without two angiographic projections at least with $>25^{\circ}$ difference couldn't be reconstructed. However, total strut and cross-section numbers provided well-fitted statistical models for getting reliable conclusions. The effect of low WSS at follow-up was not evaluated in the present study, whereas such clinical inferences can be exemplified from the literature.

\section{Conclusion}

Treated plaque type influences strut embedment/protrusion in the vessel wall. OCT has high accuracy to detect calcified plaques with its circumferential extent and depth, which are crucial information for lesion preparation such as balloon pre-dilatation, debulking, etc. Shear stress distribution is related with strut penetration in the vessel wall. Following BRS implantation, embedment/protrusion analysis may help to improve implantation process and local haemodynamic forces, which may potentially influence neointimal hyperplasia and thrombus formation.
Conflict of interest: P.W.S. is a member of the International Advisory Board of Abbott Vascular. Y.O. is a member of the International Advisory Board of Abbott Vascular. E.T. has a research grant from TUBITAK (The Research and Scientific Council of Turkey). All other authors declared no conflict of interest.

\section{References}

1. Serruys PW, Chevalier B, Sotomi Y, Cequier A, Carrie D, Piek JJ et al. Comparison of an everolimus-eluting bioresorbable scaffold with an everolimuseluting metallic stent for the treatment of coronary artery stenosis (ABSORB II): a 3 year, randomised, controlled, single-blind, multicentre clinical trial. Lancet 2016;388:2479-91.

2. Collet C, Asano T, Miyazaki Y, Tenekecioglu E, Katagiri Y, Sotomi Y et al. Late thrombotic events after bioresorbable scaffold implantation: a systematic review and meta-analysis of randomized clinical trials. Eur Heart J 2017;38:2559-66.

3. Suwannasom P, Sotomi Y, Ishibashi Y, Cavalcante R, Albuquerque FN, Macaya C et al. The impact of post-procedural asymmetry, expansion, and eccentricity of bioresorbable everolimus-eluting scaffold and metallic everolimus-eluting stent on clinical outcomes in the ABSORB II trial. JACC Cardiovasc Interv 2016;9: 1231-42.

4. Sotomi Y, Suwannasom P, Tenekecioglu E, Collet C, Nakatani S, Okamura T et al. Imaging assessment of bioresorbable vascular scaffolds. Cardiovasc Interv Ther 2018;33:11-22.

5. Ng J, Bourantas CV, Torii R, Ang HY, Tenekecioglu E, Serruys PW et al. Local hemodynamic forces after stenting: implications on restenosis. Arterioscler Thromb Vasc Biol 2017;37:2231-42.

6. Tenekecioglu E, Torii R, Sotomi Y, Collet C, Dijkstra J, Miyazaki Y et al. The effect of strut protrusion on shear stress distribution: hemodynamic insights from a prospective clinical trial. JACC Cardiovasc Interv 2017;10:1803-5.

7. Kini AS, Vengrenyuk Y, Pena J, Motoyama S, Feig JE, Meelu OA et al. Optical coherence tomography assessment of the mechanistic effects of rotational and orbital atherectomy in severely calcified coronary lesions. Catheter Cardiovasc Interv 2015;86:1024-32.

8. Sotomi Y, Tateishi H, Suwannasom P, Dijkstra J, Eggermont J, Liu S et al. Quantitative assessment of the stent/scaffold strut embedment analysis by optical coherence tomography. Int J Cardiovasc Imaging 2016;32:871-83.

9. Sotomi Y, Onuma Y, Dijkstra J, Eggermont J, Liu S, Tenekecioglu E et al. Impact of implantation technique and plaque morphology on strut embedment and scaffold expansion of polylactide bioresorbable scaffold-insights from ABSORB Japan trial. Circ J 2016;80:2317-26.

10. Mattesini A, Secco GG, Dall'Ara G, Ghione M, Rama-Merchan JC, Lupi A et al ABSORB biodegradable stents versus second-generation metal stents: a comparison study of 100 complex lesions treated under OCT guidance. JACC Cardiovasc Interv 2014;7:741-50.

11. Serruys P, De Jaegere P, Bertrand M, Kober G, Marquis JF, Piessens J et al. Morphologic change in coronary artery stenosis with the Medtronic Wiktor 
stent: initial results from the core laboratory for quantitative angiography. Cathet Cardiovasc Diagn 1991;24:237-45.

12. de Jaegere P, Serruys PW, van Es GA, Bertrand M, Wiegand V, Marquis JF et al. Recoil following Wiktor stent implantation for restenotic lesions of coronary arteries. Cathet Cardiovasc Diagn 1994;32:147-56.

13. Sotomi $Y$, Onuma $Y$, Suwannasom P, Tateishi H, Tenekecioglu E, Zeng $Y$ et al. Is quantitative coronary angiography reliable in assessing the lumen gain after treatment with the everolimus-eluting bioresorbable polylactide scaffold? Eurolntervention 2016;12:e998-e1008.

14. Bourantas CV, Papafaklis MI, Lakkas L, Sakellarios A, Onuma Y, Zhang YJ et al. Fusion of optical coherence tomographic and angiographic data for more accurate evaluation of the endothelial shear stress patterns and neointimal distribution after bioresorbable scaffold implantation: comparison with intravascular ultrasound-derived reconstructions. Int J Cardiovasc Imaging 2014;30:485-94.

15. Nakatani S, Sotomi Y, Ishibashi Y, Grundeken MJ, Tateishi H, Tenekecioglu E et al. Comparative analysis method of permanent metallic stents (XIENCE) and bioresorbable poly-L-lactic (PLLA) scaffolds (Absorb) on optical coherence tomography at baseline and follow-up. Eurolntervention 2016;12:1498-509.

16. Serruys PW, Onuma Y, Ormiston JA, de Bruyne B, Regar E, Dudek D et al. Evaluation of the second generation of a bioresorbable everolimus drug-eluting vascular scaffold for treatment of de novo coronary artery stenosis: six-month clinical and imaging outcomes. Circulation 2010;122:2301-12.

17. Papafaklis MI, Bourantas CV, Theodorakis PE, Katsouras CS, Naka KK, Fotiadis $\mathrm{DI}$ et al. The effect of shear stress on neointimal response following sirolimusand paclitaxel-eluting stent implantation compared with bare-metal stents in humans. JACC Cardiovasc Interv 2010;3:1181-9.

18. Bourantas CV, Papafaklis MI, Kotsia A, Farooq V, Muramatsu T, Gomez-Lara J et al. Effect of the endothelial shear stress patterns on neointimal proliferation following drug-eluting bioresorbable vascular scaffold implantation: an optical coherence tomography study. JACC Cardiovasc Interv 2014;7:315-24.

19. Stone PH, Saito S, Takahashi S, Makita Y, Nakamura S, Kawasaki T et al. Prediction of progression of coronary artery disease and clinical outcomes using vascular profiling of endothelial shear stress and arterial plaque characteristics: the PREDICTION Study. Circulation 2012;126:172-81.

20. Carew TE, Vaishnav RN, Patel DJ. Compressibility of the arterial wall. Circ Res 1968;23:61-8.

21. Chuong C], Fung YC. Compressibility and constitutive equation of arterial wall in radial compression experiments. J Biomech 1984;17:35-40.

22. Sadat U, Teng Z, Gillard JH. Biomechanical structural stresses of atherosclerotic plaques. Expert Rev Cardiovasc Ther 2010;8:1469-81.

23. Timmins LH, Meyer CA, Moreno MR, Moore JE. Effects of stent design and atherosclerotic plaque composition on arterial wall biomechanics. J Endovasc Ther 2008;15:643-54.

24. Salunke NV, Topoleski LD, Humphrey JD, Mergner WJ. Compressive stressrelaxation of human atherosclerotic plaque. J Biomed Mater Res 2001;55:236-41.

25. Ohayon J, Mesnier N, Broisat A, Toczek J, Riou L, Tracqui P. Elucidating atherosclerotic vulnerable plaque rupture by modeling cross substitution of ApoE-/- mouse and human plaque components stiffnesses. Biomech Model Mechanobiol 2012;11:801-13.

26. Hayashi K. Experimental approaches on measuring the mechanical properties and constitutive laws of arterial walls. J Biomech Eng 1993;115:481-8.

27. Koniari I, Mavrilas D, Papadaki H, Karanikolas M, Mandellou M, Papalois A et al. Structural and biomechanical alterations in rabbit thoracic aortas are associated with the progression of atherosclerosis. Lipids Health Dis 2011;10:125.

28. Farrar DJ, Riley WA, Bond MG, Barnes RN, Love LA. Detection of early atherosclerosis in $M$. fascicularis with transcutaneous ultrasonic measurement of the elastic properties of the common carotid artery. Tex Heart Inst J 1982;9:335-43.

29. Weissman NJ, Palacios IF, Weyman AE. Dynamic expansion of the coronary arteries: implications for intravascular ultrasound measurements. Am Heart J 1995;130:46-51.

30. Pericevic I, Lally C, Toner D, Kelly DJ. The influence of plaque composition on underlying arterial wall stress during stent expansion: the case for lesion-specific stents. Med Eng Phys 2009;31:428-33.

31. Tang D, Yang C, Zheng J, Woodard PK, Saffitz JE, Sicard GA et al. Quantifying effects of plaque structure and material properties on stress distributions in human atherosclerotic plaques using 3D FSI models. J Biomech Eng 2005;127: 1185-94.

32. Sprague EA, Luo J, Palmaz JC. Endothelial cell migration onto metal stent surfaces under static and flow conditions. J Long Term Eff Med Implants 2000;10:97-110.

33. Tenekecioglu E, Bourantas CV, Onuma Y, Serruys PW. Sealing of calcified plaques after bioresorbable scaffold implantations: a five-year follow up. Int J Cardiovasc Imaging 2017;33:451-2.

34. Chatzizisis YS, Coskun AU, Jonas M, Edelman ER, Feldman CL, Stone PH. Role of endothelial shear stress in the natural history of coronary atherosclerosis and vascular remodeling: molecular, cellular, and vascular behavior. J Am Coll Cardiol 2007;49:2379-93.

35. Phelps JE, DePaola N. Spatial variations in endothelial barrier function in disturbed flows in vitro. Am J Physiol Heart Circ Physiol 2000;278:H469-76.

36. Foin N, Gutierrez-Chico JL, Nakatani S, Torii R, Bourantas CV, Sen S et al. Incomplete stent apposition causes high shear flow disturbances and delay in neointimal coverage as a function of strut to wall detachment distance: implications for the management of incomplete stent apposition. Circ Cardiovasc Interv 2014;7:180-9.

37. Tenekecioglu E, Torii R, Bourantas C, Crake T, Zeng Y, Sotomi Y et al. Preclinical assessment of the endothelial shear stress in porcine-based models following implantation of two different bioresorbable scaffolds: effect of scaffold design on the local haemodynamic micro-environment. Eurolntervention 2016;12 1296.

38. Tenekecioglu E, Torii R, Bourantas C, Sotomi Y, Cavalcante R, Zeng Y et al. Difference in haemodynamic microenvironment in vessels scaffolded with absorb BVS and mirage BRMS: insights from a preclinical endothelial shear stress study. Eurolntervention 2017;13:1327-35.

39. Tenekecioglu E, Poon EK, Collet C, Thondapu V, Torii R, Bourantas CV et al. The nidus for possible thrombus formation: insight from the microenvironment of bioresorbable vascular scaffold. JACC Cardiovasc Interv 2016;9:2167-8. 